\title{
Characterizing new physics with polarized beams at high-energy hadron colliders
}

\author{
Benjamin Fuks, ${ }^{a, b}$ Josselin Proudom, $^{c}$ Juan Rojo ${ }^{a}$ and Ingo Schienbein ${ }^{c}$ \\ ${ }^{a}$ Theory Division, Physics Department, CERN, \\ CH-1211 Geneva 23, Switzerland \\ ${ }^{b}$ Institut Pluridisciplinaire Hubert Curien/Département Recherches Subatomiques, \\ Université de Strasbourg/CNRS-IN2P3, 23 rue du Loess, F-67037 Strasbourg, France \\ ${ }^{c}$ Laboratoire de Physique Subatomique et de Cosmologie, \\ Université Grenoble-Alpes, CNRS/IN2P3, 53 avenue des Martyrs, 38026 Grenoble, France \\ E-mail: benjamin.fuks@cern.ch, josselin.proudom@lpsc.in2p3.fr, \\ juan.rojo@cern.ch, ingo.schienbein@lpsc.in2p3.fr
}

ABstract: The TeV energy region is currently being explored by both the ATLAS and CMS experiments of the Large Hadron Collider and phenomena beyond the Standard Model are extensively searched for. Large fractions of the parameter space of many models have already been excluded, and the ranges covered by the searches will certainly be increased by the upcoming energy and luminosity upgrades. If new physics has to be discovered in the forthcoming years, the ultimate goal of the high-energy physics program will consist of fully characterizing the newly-discovered degrees of freedom in terms of properties such as their masses, spins and couplings. The scope of this paper is to show how the availability of polarized beams at high-energy proton-proton colliders could yield a unique discriminating power between different beyond the Standard Model scenarios. We first discuss in a model-independent way how this discriminating power arises from the differences between polarized and unpolarized parton distribution functions. We then demonstrate how polarized beams allow one not only to disentangle different production mechanisms giving the same final-state signature, but also to obtain information on the parameters of the hypothetical new physics sector of the theory. This is illustrated in the case of a particular class of scenarios leading to monotop production. We consider three specific models that could produce a monotop signature in unpolarized proton collisions, and show how they could be distinguished by means of single- and double-spin asymmetries in polarized collisions. Our results are presented for both the Large Hadron Collider operating at a center-of-mass energy of $14 \mathrm{TeV}$ and a recently proposed Future Circular Collider assumed to collide protons at a center-of-mass energy of $100 \mathrm{TeV}$.

Keywords: QCD Phenomenology, Hadronic Colliders

ArXiv EPRINT: 1403.2383 


\section{Contents}

1 Introduction 1

2 Spin asymmetries at polarized hadron colliders 2

3 Pinning down monotop production dynamics with polarized beams $\quad 9$

$\begin{array}{lll}3.1 & \text { Monotop production in the RPV MSSM } & 10\end{array}$

$\begin{array}{lll}3.2 & \text { Other scenarios for monotop production } & 13\end{array}$

$\begin{array}{lll}3.3 & \text { Impact of monotop charge tagging } & 17\end{array}$

$\begin{array}{lll}3.4 & \text { Summary } & 17\end{array}$

4 Conclusions and outlook $\quad 17$

\section{Introduction}

After three years of data-taking, the ATLAS and CMS experiments have already probed quite extensively the $\mathrm{TeV}$ scale. With the upcoming proton-proton runs at $13 \mathrm{TeV}$ and $14 \mathrm{TeV}$ and the proposed high-luminosity upgrade of the Large Hadron Collider (LHC), searches for new phenomena, particles and interactions promise to survey an even wider portion of the parameter space of a huge variety of beyond the Standard Model (BSM) scenarios. In most of the studies, the relevant experimental analyses are motivated by theoretical arguments, implying some key new physics final-state signatures that should be looked for. However, those signatures are neither typical of a given theory, nor of a given benchmark scenario of a specific model. One of the most famous examples illustrating this fact arises from the Minimal Supersymmetric Standard Model (MSSM) [1, 2] and Universal Extra Dimensions models [3], which both predict the pair production of Standard Model partners followed by their cascade decay into a final state enriched in charged leptons and jets, and containing in addition a large amount of missing transverse energy. Consequently, beyond discovery, the task of disentangling BSM theories (and even different scenarios within a specific theory) that share a common final-state signature is known to be far from trivial.

Additionally to the LHC, there is another high-energy hadron collider which is providing an impressive wealth of results. The RHIC collider at the Brookhaven National Laboratory has successfully operated in its polarized proton-proton mode at $200 \mathrm{GeV}$ and $500 \mathrm{GeV}$, collecting data with an integrated luminosity of more than $1 \mathrm{fb}^{-1}$. Although these polarized collisions are mainly dedicated to spin physics, pioneering BSM studies have shown the non-negligible impact of beam polarization to get a handle on (some of) the model parameters of specific theories [4-10]. Another interesting possibility that has been put forward was the study of anomalous $W W \gamma$ and $W W Z$ couplings in a polarized 
upgrade of the Tevatron collider [11]. In addition to the existing RHIC polarized proton collider, most of the aforementioned studies have also considered possible polarization upgrades of both the Tevatron and the LHC. However, although those upgrades have been already discussed in the past and are perfectly feasible $[12,13]$, they are quite unlikely to be realized.

In contrast, first discussions on a Future Circular Collider (FCC) with a center-of-mass energy of $100 \mathrm{TeV}$ are now starting. Therefore, this is the right time to begin to present the physics cases motivating different operating options of such a machine, including a possible polarized mode. This paper lies in this context, and intends to show how polarized proton beams colliding at $14 \mathrm{TeV}$ and $100 \mathrm{TeV}$ could provide an interesting way of disentangling new physics models featuring the same final-state signature. The key ingredient that underlies this idea is the difference between polarized and unpolarized parton distribution functions (PDFs) for a given flavor, that leads to quite different spin asymmetries in polarized collisions depending on the initial-state partonic production channel. This thus allows one to distinguish different BSM physics scenarios which, characterized by different production mechanism, provide the same final-state signature in unpolarized collisions.

For the sake of illustration, we focus on the investigation of the recently proposed monotop signature $[14,15]$, which corresponds to the production of a single top in association with missing transverse energy and no other particle. Monotops naturally appear in several extensions of the Standard Model, like for example in supersymmetric theories with $R$-parity violation (RPV) where they are issued from the decay of a singly-produced top squark [16-19]. Besides this RPV mode, monotops can also be produced in various dark matter models [20-23] where the monotop state originates either from the decay of a vector resonance, or from tree-level flavor-changing neutral interactions with a particle giving rise to missing energy. In the following, we discuss how the measurements of singlespin and double-spin asymmetries at a polarized LHC or at a polarized FCC would allow one to get additional information on the nature of the initial partons at the origin of the monotop signal and show how this could be used in order to constrain the underlying new physics scenario.

The paper is organized as follows: in section 2, we perform a detailed study of parton densities and parton luminosities in the framework of polarized proton-proton collisions at center-of-mass energies of $14 \mathrm{TeV}$ and $100 \mathrm{TeV}$, showing the strength of spin asymmetries to discriminate among different initial states. Then, we assume the observation of a monotop excess in unpolarized proton-proton collisions and illustrate in section 3 how spin asymmetries possibly allow one to get information on the new physics scenarios that have yielded the signal. Our conclusions are presented in section 4.

\section{Spin asymmetries at polarized hadron colliders}

As has been mentioned in the introduction, polarized beams at high-energy hadron colliders would provide a unique opportunity to characterize any new physics signal that might have been previously observed in unpolarized collisions. This appealing possibility relies on the fact that polarized and unpolarized parton-parton luminosities show quite different 
behaviors for a given flavor combination. Therefore, single- and double-spin asymmetries in polarized hadron collisions can provide information on the initial partonic state of any given process, thus allowing one to disentangle different beyond the Standard Model production scenarios that lead to the same final state signatures.

In the next section we will exploit these remarkable properties to distinguish between new physics scenarios for monotop production at the LHC, characterized by different initial state production mechanisms and thus by different single- and double-spin asymmetries in polarized collisions. However, before discussing specific models, it is instructive to first evaluate a variety of single- and double-spin asymmetries at the level of parton luminosities rather than at the full hadronic cross section level. This approximation is useful since in many cases of interest the polarized and unpolarized matrix elements are similar, and thus the spin asymmetries computed from the partonic luminosities only already carry the bulk of the relevant physics which is accessible experimentally via the hadron-level asymmetries.

First of all we compare polarized and unpolarized PDFs. To fix the notation, we define unpolarized and polarized parton distributions as usual,

$$
\begin{aligned}
q_{i}\left(x, Q^{2}\right) & \equiv q_{i}^{\uparrow}\left(x, Q^{2}\right)+q_{i}^{\downarrow}\left(x, Q^{2}\right), \\
\Delta q_{i}\left(x, Q^{2}\right) & \equiv q_{i}^{\uparrow}\left(x, Q^{2}\right)-q_{i}^{\downarrow}\left(x, Q^{2}\right),
\end{aligned}
$$

in terms of the two different possible longitudinal polarization states of partons within the nucleon,

$$
\begin{aligned}
& q_{i}^{\uparrow}\left(x, Q^{2}\right)=q_{i}\left(x, Q^{2}\right)+\Delta q_{i}\left(x, Q^{2}\right), \\
& q_{i}^{\downarrow}\left(x, Q^{2}\right)=q_{i}\left(x, Q^{2}\right)-\Delta q_{i}\left(x, Q^{2}\right) .
\end{aligned}
$$

In figure 1, we present a comparison between the different PDFs of the most updated unpolarized and polarized sets from the NNPDF Collaboration, ${ }^{1}$ NNPDF2.3 [24] and NNPDFpol1.1 $[25,26]$ respectively. The various PDFs have been evaluated at a typical hadron collider scale of $Q^{2}=10^{4} \mathrm{GeV}^{2}$ using the LHAPDF interface [27].

There are various interesting features to remark in figure 1 . The first one is that polarized PDFs are always smaller (in absolute value) than their unpolarized counterparts, a consequence of the positivity condition of polarized PDFs [28], which at Born level reads,

$$
\left|\Delta q_{i}\left(x, Q^{2}\right)\right| \leq q_{i}\left(x, Q^{2}\right)
$$

At the next-to-leading order, similar relations hold but only for physical observables like polarized structure functions. The second feature is that at small- $x$ the growth of the polarized PDFs $x \Delta q_{i}\left(x, Q^{2}\right)$ is largely suppressed with respect to that of the unpolarized ones $x q_{i}\left(x, Q^{2}\right)[29]$. As will be shown below, these two features have the important implication that spin asymmetries will be sizable and thus experimentally accessible only for final states with large invariant masses. This indeed probes the polarized PDFs at medium

\footnotetext{
${ }^{1}$ The polarized set of parton densities NNPDFpol1.1 is available from the webpage https://nnpdf .hepforge.org/html/nnpdfpol10/nnpdfpol10sets.html.
} 

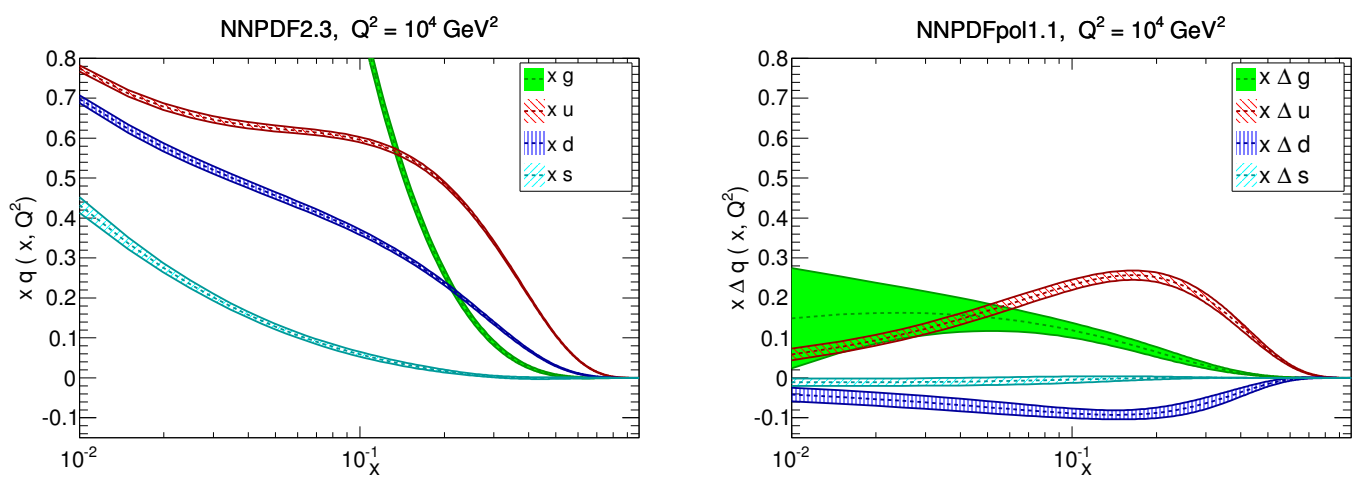

Figure 1. Comparison between unpolarized (left) and polarized (right) up, down, strange and gluon PDFs from the most updated sets of the NNPDF family, NNPDF2.3 and NNPDFpol1.1 respectively. PDFs have been evaluated at a typical high-energy hadron collider scale of $Q^{2}=10^{4} \mathrm{GeV}^{2}$.

and large values of $x$, two regions where their magnitude is comparable to the one of the unpolarized parton densities.

In addition, and this is of particular importance for the problem at hand, a specific flavor leads to different qualitative behaviors for the polarized and unpolarized PDFs. For instance, $\Delta u$ and $\Delta d$ have the opposite sign, while $u$ and $d$ have both the same sign and the same shape. This will translate into qualitatively different behaviors for the various spin asymmetries depending on the underlying initial partonic state.

After comparing PDFs at the unpolarized and polarized level, we move to the study of partonic luminosities [30] and the corresponding single- and double-spin asymmetries. We define the partonic luminosity for the scattering of two partons $i$ and $j$ in unpolarized hadronic collisions, leading to a final state of mass $m_{X}$, as

$$
\mathcal{L}_{i j}=\frac{1}{S} \int_{\tau}^{1} \frac{d x}{x} \frac{1}{1+\delta_{i j}}\left[q_{i}\left(x, m_{X}\right) q_{j}\left(\frac{\tau}{x}, m_{X}\right)+q_{i}\left(\frac{\tau}{x}, m_{X}\right) q_{j}\left(x, m_{X}\right)\right],
$$

where the $\delta_{i j}$ factor removes the double counting in the case of a same PDF combination $i=$ $j$, and the collider center-of-mass energy squared $S=E_{\mathrm{cm}}^{2}$ enters through the variable $\tau=$ $\hat{s} / S$. We can also define corresponding quantities involving polarized parton distributions and thus relevant for polarized collisions. The partonic luminosity relevant for single-spin asymmetries is defined as

$$
\mathcal{L}_{i j}^{L}=\frac{1}{S} \int_{\tau}^{1} \frac{d x}{x} \frac{1}{1+\delta_{i j}}\left[q_{i}\left(x, m_{X}\right) \Delta q_{j}\left(\frac{\tau}{x}, m_{X}\right)+q_{i}\left(\frac{\tau}{x}, m_{X}\right) \Delta q_{j}\left(x, m_{X}\right)\right],
$$

while for double-spin asymmetries, we use

$$
\mathcal{L}_{i j}^{L L}=\frac{1}{S} \int_{\tau}^{1} \frac{d x}{x} \frac{1}{1+\delta_{i j}}\left[\Delta q_{i}\left(x, m_{X}\right) \Delta q_{j}\left(\frac{\tau}{x}, m_{X}\right)+\Delta q_{i}\left(\frac{\tau}{x}, m_{X}\right) \Delta q_{j}\left(x, m_{X}\right)\right]
$$

In our notation, the $L$ and $L L$ superscripts indicate that these luminosities enter the description of single- and double-spin asymmetries in polarized collisions, respectively. 
We now define unpolarized and polarized hadron-level cross sections by

$$
\begin{aligned}
\sigma_{0} & =\frac{1}{4}\left[\sigma^{\uparrow \uparrow}+\sigma^{\downarrow \downarrow}+\sigma^{\uparrow \downarrow}+\sigma^{\downarrow \uparrow}\right], \\
\sigma_{L} & =\frac{1}{4}\left[\sigma^{\uparrow \uparrow}-\sigma^{\downarrow \downarrow}-\sigma^{\uparrow \downarrow}+\sigma^{\downarrow \uparrow}\right], \\
\sigma_{L L} & =\frac{1}{4}\left[\sigma^{\uparrow \uparrow}+\sigma^{\downarrow \downarrow}-\sigma^{\uparrow \downarrow}-\sigma^{\downarrow \uparrow}\right] .
\end{aligned}
$$

Here $\sigma_{0}$ stands for the unpolarized cross sections and $\sigma_{L}$ and $\sigma_{L L}$ for singly and doublypolarized cross sections, respectively, where an up-arrow denotes a helicity $h=+1$ and a down-arrow a helicity $h=-1$ of longitudinally polarized hadrons in the initial state. We recall that in the case of singly-polarized cross sections, only one of the hadrons (the second one here) is polarized. It is useful to consider ratios of these cross sections, or spin asymmetries, since experimental uncertainties cancel to a good degree. If a single beam is polarized, the experimentally relevant quantity is the single-spin asymmetry, defined as

$$
A_{L}=\frac{\sigma_{L}}{\sigma_{0}},
$$

whereas if both beams are polarized, the relevant quantity is the double-spin asymmetry

$$
A_{L L}=\frac{\sigma_{L L}}{\sigma_{0}} .
$$

Eqs. (2.9)-(2.11) define experimentally accessible observables since they are expressed in terms of polarized hadrons. In order to compare data with theoretical predictions, in perturbative QCD the factorization theorem allows one to write hadronic cross sections as convolutions of parton distribution functions with parton level cross sections,

$$
\begin{aligned}
\sigma_{0} & =q_{i} \otimes q_{j} \otimes \hat{\sigma}_{0, i j}=\mathcal{L}_{i j} \otimes\left[\hat{s} \hat{\sigma}_{0, i j}\right] \\
\sigma_{L} & =q_{i} \otimes \Delta q_{j} \otimes \hat{\sigma}_{L, i j}=\mathcal{L}_{i j}^{L} \otimes\left[\hat{s} \hat{\sigma}_{L, i j}\right], \\
\sigma_{L L} & =\Delta q_{i} \otimes \Delta q_{j} \otimes \hat{\sigma}_{L L, i j}=\mathcal{L}_{i j}^{L L} \otimes\left[\hat{s} \hat{\sigma}_{L L, i j}\right] .
\end{aligned}
$$

The polarized partonic cross sections are here defined in complete analogy to the polarized hadron-level expressions of eqs. (2.9)-(2.11), namely

$$
\begin{aligned}
\hat{\sigma}_{0} & =\frac{1}{4}\left[\hat{\sigma}^{\uparrow \uparrow}+\hat{\sigma}^{\downarrow \downarrow}+\hat{\sigma}^{\uparrow \downarrow}+\hat{\sigma}^{\downarrow \uparrow}\right], \\
\hat{\sigma}_{L} & =\frac{1}{4}\left[\hat{\sigma}^{\uparrow \uparrow}-\hat{\sigma}^{\downarrow \downarrow}-\hat{\sigma}^{\uparrow \downarrow}+\hat{\sigma}^{\downarrow \uparrow}\right], \\
\hat{\sigma}_{L L} & =\frac{1}{4}\left[\hat{\sigma}^{\uparrow \uparrow}+\hat{\sigma}^{\downarrow \downarrow}-\hat{\sigma}^{\uparrow \downarrow}-\hat{\sigma}^{\downarrow \uparrow}\right],
\end{aligned}
$$

where now the helicities are those of the incoming quarks and gluons in the partonic collision. Furthermore, a sum over all relevant partonic subprocesses is implied (i.e., over $i, j)$ and we refer to eqs. (2.6)-(2.8) for the definition of the partonic luminosities.

For many cases of physical interest, the expressions in eqs. (2.14)-(2.16) and consequently the asymmetries in eqs. (2.12) and (2.13) can be further simplified. Firstly, the 
dimensionless cross sections $\hat{s} \hat{\sigma}_{i j}$ are often either constant far above the production threshold (see, e.g., figure 70 in ref. [30]) or, in the case of a narrow $s$-channel resonance, they are peaked at threshold, that is, $\hat{s} \simeq m_{X}^{2}$. In the latter case, we end up having simple expressions of the hadron-level asymmetries in terms of (ratios of weighted sums of) parton luminosities. Secondly, the absolute values of the polarized and unpolarized parton-level matrix elements are often the same or very similar, leading to further simplifications. In cases where there is a single dominant particular sub-channel, the hadronic asymmetries are just simple ratios of parton luminosities, as can be deduced from the single-spin and double-spin asymmetries of eqs. (2.14)-(2.16),

$$
A_{L}^{i j}=\frac{\mathcal{L}_{i j}^{L}}{\mathcal{L}_{i j}} \quad \text { and } \quad A_{L L}^{i j}=\frac{\mathcal{L}_{i j}^{L L}}{\mathcal{L}_{i j}}
$$

In the rest of this section, we focus on results for single and double-spin asymmetries computed from eq. (2.20) for different initial state partonic sub-channels. We have calculated these asymmetries for the LHC collider operating at a center-of-mass energy of $14 \mathrm{TeV}$ (LHC $14 \mathrm{TeV}$ ), assuming a possible future polarized upgrade, as well as for the polarized mode of an hypothetical Future Circular Collider with a center-of-mass energy of $100 \mathrm{TeV}$ (FCC $100 \mathrm{TeV}$ ). As polarized PDFs we use the NNPDFpol1.1 [25, 26] and DSSV08 [31] sets, together with the corresponding unpolarized counterparts, NNPDF2.3 [24] and MRST01 [32]. Comparing the predictions of NNPDFpol1.1 with those of DSSV08 is useful in order to verify which features of the spin asymmetries are generic irrespective of the specific details of the particular polarized PDF set used.

It is clear from the definition of eqs. (2.6)-(2.8) that to first approximation, luminosities are invariant if the center-of-mass energy is modified, $\sqrt{S^{\prime}}=k \sqrt{S}$, provided that the final state mass is also modified in the same way, $m_{X}^{\prime}=k m_{X}$, since in this case the variable $\tau$ is invariant. However, logarithmic corrections to the DGLAP evolution of the PDFs modify this picture, though they should not change any qualitative conclusion. This property will be explicitly verified below when comparing the spin asymmetries at LHC $14 \mathrm{TeV}$ and at FCC $100 \mathrm{TeV}$.

First of all, we compare the single-spin asymmetries at $\mathrm{LHC} 14 \mathrm{TeV}$ for the production of a final state with invariant mass $m_{X}$ assuming different partonic initial states. We compare the consistency of the asymmetries obtained with NNPDF with those obtained with DSSV/MRST. In all cases, the uncertainty band on the asymmetries corresponds to that of the polarized PDFs, since in this respect the unpolarized PDF uncertainties can be neglected. We show the asymmetries for $g g$, $u u$ and $d d$ initial states in the upper row of figure 2 and for the $d s, d b$ and $s b$ initial states in the bottom row of the figure. The DSSV08 densities consist of a PDF set obtained in the fixed-flavor-number scheme, and therefore the polarized bottom $\mathrm{PDF} \Delta b=\Delta \bar{b}=0$. While differences between fixed-flavornumber and variable-flavor-number schemes lead to substantial differences for unpolarized PDFs [33], this is considered less important for polarized PDFs in the region with available experimental data where the contribution from heavy quarks is small. However, this is no longer true when evolving upwards in $Q^{2}$ to the region relevant for collider physics, where heavy quark PDFs are not negligible even in the polarized case. 

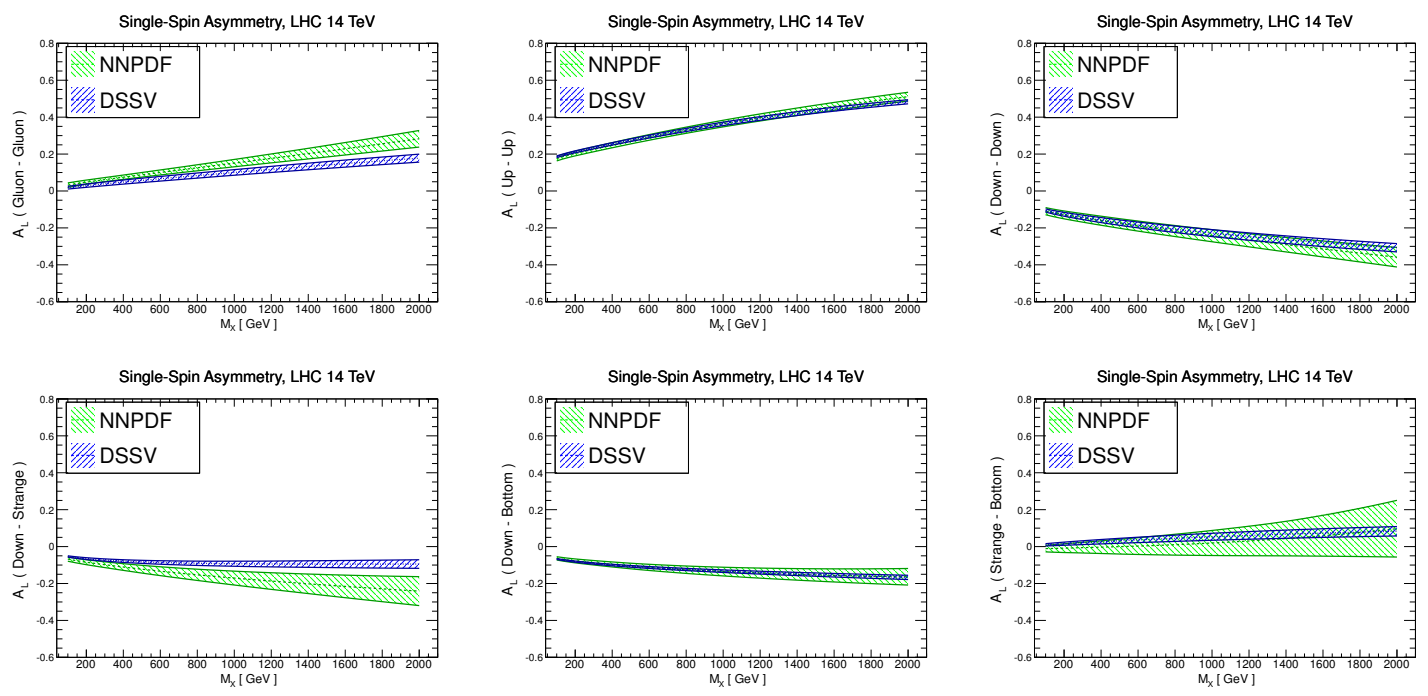

Figure 2. The single-spin asymmetry $A_{L}$ at the parton luminosity level, that we compute from eq. (2.20), at LHC $14 \mathrm{TeV}$, and for various initial-state partonic combinations. We compare results obtained using NNPDFpol1.1/NNPDF2.3 with those obtained using DSSV/MRST and present them as function of the invariant mass of the final state $m_{X}$. The bands correspond to the polarized PDF uncertainties.
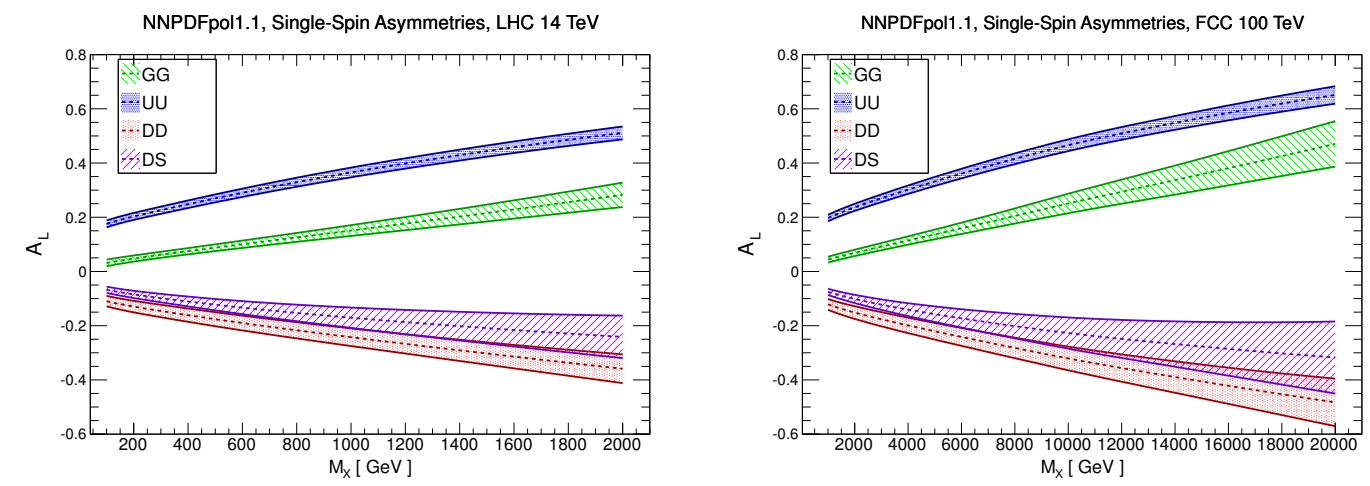

Figure 3. Summary of the single-spin asymmetries $A_{L}$ for a variety of initial state partonic combinations as a function of the invariant mass of the produced final state $m_{X}$ at the LHC $14 \mathrm{TeV}$ (left panel) and at an FCC $100 \mathrm{TeV}$ (right panel). The asymmetries have been obtained using NNPDFpol1.1/NNPDF2.3.

In general there is a reasonable qualitative agreement between the results from NNPDFpol1.1 and those of DSSV, with some quantitative differences, for instance in asymmetries that involve the polarized strange PDF. This is expected since NNPDFpol1.1 and DSSV08 generally agree well for all PDFs but for $\Delta s\left(x, Q^{2}\right)$, where even the sign is opposite [33]. Larger PDF uncertainties are obtained using NNPDFpol1.1, partially due to the more flexible functional form of the input PDFs as compared to DSSV08. Results for the single spin asymmetries for a $100 \mathrm{TeV}$ FCC are qualitatively similar once the value of the final state mass is properly rescaled as discussed above, so that results are not shown explicitly.

Results for the single-spin asymmetries in the $g g, u u, d d$ and $d s$ partonic sub-channels 



Figure 4. The double-spin asymmetry $A_{L L}$ at the PDF level, eq. (2.20), at LHC $14 \mathrm{TeV}$ for various initial-state partonic combinations, comparing the results obtained using NNPDFpol1.1/NNPDF2.3 with those obtained using DSSV/MRST.

for $\mathrm{LHC} 14 \mathrm{TeV}$ and FCC $100 \mathrm{TeV}$ are summarized in figure 3. It is apparent that the property which we have discussed above, namely that if the final state mass range is suitably scaled, the qualitative features of the spin asymmetries are the same at center-ofmass energies of $14 \mathrm{TeV}$ and $100 \mathrm{TeV}$. The most striking observed property is that different partonic sub-channels lead to very different asymmetries. In this particular case, just a measurement of the sign of the asymmetry would indicate which are the dominant partonic initial states, and measurements of $A_{\mathrm{L}}$ with a few percent experimental uncertainty would even distinguish between $g g$ and $q q$ initiated final states.

Double-spin asymmetries are experimentally more challenging since their absolute values are smaller. The reason for this is because they involve the convolution of two polarized PDFs in the numerator, instead of just one as for single-spin asymmetries. The results for various partonic channels are summarized in figure 4. Again reasonable agreement between NNPDFpol1.1 and DSSV08 is found. Asymmetries involving quarks are larger than those involving gluons, reflecting that the $\Delta q$ densities are larger than the $\Delta g$ one at large- $x$, as shown in figure 1. Moreover, large final state masses are required to yield spin asymmetries that are larger than a few percent.

The final comparison is provided by double-spin asymmetry calculations for the $g g$, $u u, d d$ and $d s$ partonic sub-channels for LHC $14 \mathrm{TeV}$ and FCC $100 \mathrm{TeV}$ and we collect the results in figure 5. The PDF uncertainties are found very large, since there is far less experimental information in the determinations of the $\Delta q_{i}$ densities than in the unpolarized case. However, if the measurement of a vanishing double-spin asymmetry could be performed, this would be a valuable piece of information since it would exclude that the final state is dominantly produced from uu scattering. Moreover, double-spin asymmetry measurements could nevertheless be used to verify the results obtained from single-spin asymmetries.

After this discussion at the PDF level only, in the next section we will present pre- 

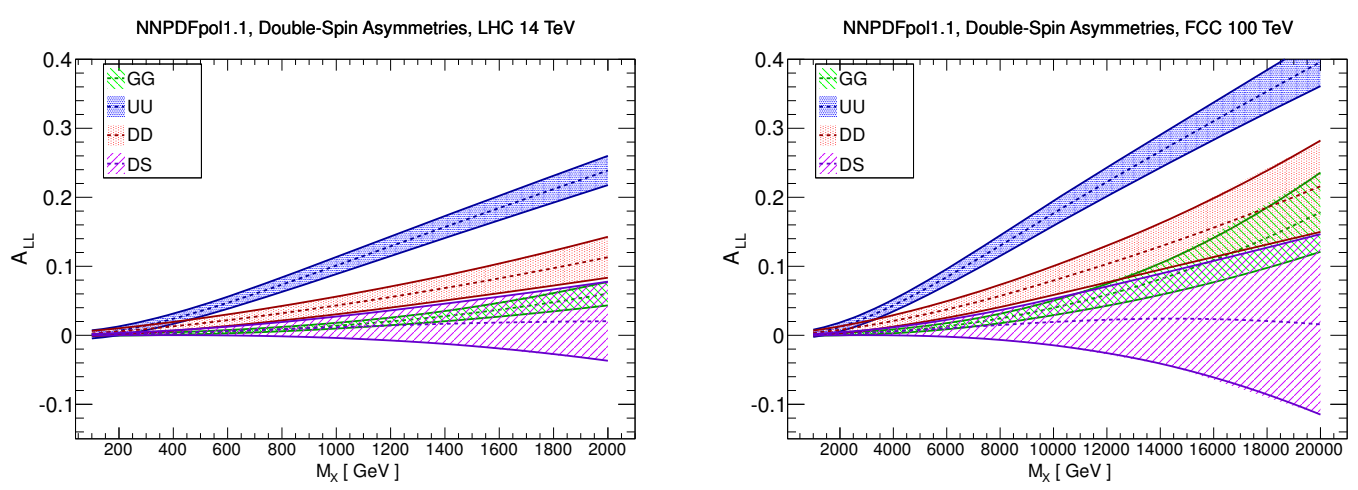

Figure 5. Summary of the double-spin asymmetries $A_{L L}$ for a variety of initial state partonic combinations as a function of the invariant mass of the produced finale state $m_{X}$ at the LHC $14 \mathrm{TeV}$ (left panel) and at an FCC $100 \mathrm{TeV}$ (right panel). The asymmetries have been obtained using NNPDFpol1.1.

dictions for hadron-level asymmetries in various scenarios for BSM monotop production. From now on we will neglect for clarity the polarized PDF uncertainties. It has already been shown in this section that they are large, however, the availability of a polarized hadron collider would also provide a large set of polarized PDF-sensitive measurements that should substantially reduce these uncertainties. In addition, in the short term, additional constraints from a variety of polarized measurements from fixed target and collider experiments like HERMES, COMPASS and RHIC will allow to further pin down the polarized PDFs. In the medium term, important constraints on polarized PDFs could also be provided by a Electron-Ion Collider (EIC) [34-37], currently under study.

\section{Pinning down monotop production dynamics with polarized beams}

In order to illustrate the power of spin asymmetries for the characterization of new physics, we focus in this work on one generic BSM signature, dubbed monotop, that has recently been proposed $[14,15]$. The monotop signature is characterized by a top quark produced singly in association with missing energy and without any additional particle. The choice of such a process to illustrate the usefulness of polarized proton-proton collisions for physics beyond the Standard Model is driven by several considerations.

First of all, the sector of the top quark is widely believed to be one of the key candidates for coupling in an enhanced way to new physics particles due to the vicinity of the top mass to the electroweak scale. Second, monotop production is negligible in the Standard Model where the top quark is produced in association with a $Z$-boson and no extra jet. This process is indeed loop-induced, GIM-suppressed and further reduced by the branching ratio of the $Z$-boson to neutrinos. This ensures that a monotop observation at the LHC (or at an FCC) can be safely thought of as a clear tell-tale sign of new physics. Finally, there exists a wide variety of new physics theories that lead to the same final-state monotop signature (see, e.g., refs. [14-23]). This process therefore offers a good way to illustrate how singlespin and double-spin asymmetries can provide a unique handle to extract information on the underlying theory realized in Nature, should monotop production be observed. 


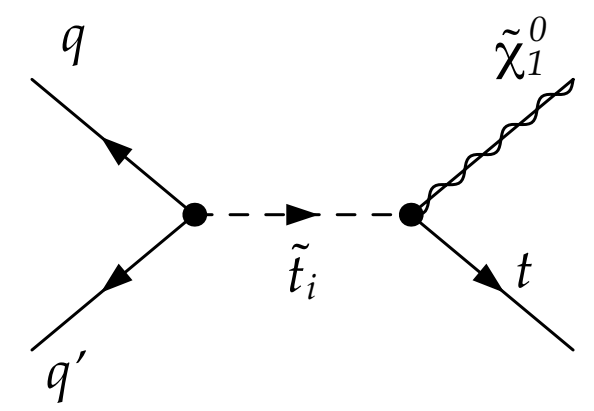

Figure 6. Feynman diagram associated with RPV monotop production. The neutralino $\tilde{\chi}_{1}^{0}$ is assumed long-lived so that it decays outside the detector, effectively giving rise to missing transverse energy. Non-resonant subprocesses have here been neglected since they are small compared to resonant production.

\subsection{Monotop production in the RPV MSSM}

We begin by considering the MSSM after supplementing its $R$-parity conserving superpotential by one single RPV operator, the so-called UDD term. As it will be shown below, this simple setup includes three distinct monotop production mechanisms hardly distinguishable in unpolarized proton-proton collisions, apart from the differences in total rates that are however dependent on unknown couplings. This contrasts with the situation when additional polarized observables are available since in this case, discriminating between the different initial states becomes possible.

We model the supersymmetric interactions among the matter sector by adding to the $R$-parity conserving MSSM superpotential $W_{\text {MSSM }}$ the RPV UDD operator,

$$
W=W_{\mathrm{MSSM}}+\frac{1}{2} \lambda_{i j k}^{\prime \prime} U_{R}^{i} D_{R}^{j} D_{R}^{k},
$$

where $U_{R}$ and $D_{R}$ are the chiral superfields associated with the up-type and down-type right-handed (s)quark supermultiplets, the color indices are implicit for clarity and the flavor indices are explicitly indicated. Monotop production is induced by non-vanishing $\lambda_{3 j k}^{\prime \prime}$ couplings together with enforcing the lightest neutralino to be long-lived, a setup almost unconstrained by experimental data [38]. If at least one of these $\lambda^{\prime \prime}$ couplings is non-vanishing, top squarks of mass $m_{\tilde{t}}$ can be resonantly produced from the scattering of two down-type antiquarks of different flavors and further decay into a top quark and a lightest neutralino (see figure 6) which, if lighter than the top quark, is long-lived enough to escape detection and gives rise to missing transverse energy in a detector [39]. The same final state could also be produced through $t / u$-channel down-type squark exchanges allowed by the interactions driven by the superpotential of eq. (3.1). We however neglect, in the following, these non-resonant contributions with respect to the resonant $s$-channel diagram, that we have explicitly verified to be largely dominant.

In the RPV supersymmetric framework described above, the fully polarized partonic cross-section for monotop production from a $\bar{q} \bar{q}^{\prime}$ initial-state is given by

$$
\hat{\sigma}_{R P V}^{h_{1} h_{2}}\left(\bar{q}_{j} \bar{q}_{k} \rightarrow t \tilde{\chi}_{1}^{0}\right)=\frac{\left(1-h_{1}\right)\left(1-h_{2}\right) \pi\left|\lambda_{3 j k}^{\prime \prime} \sin \theta_{\tilde{t}}\right|^{2}}{6} \operatorname{BR}\left(\tilde{t} \rightarrow t \tilde{\chi}_{1}^{0}\right) \delta\left(\hat{s}-m_{\tilde{t}}^{2}\right),
$$




\begin{tabular}{|c|c|c|c|}
\hline Scenario & $\sigma_{0}$ & $\sigma_{L}$ & $\sigma_{L L}$ \\
\hline \multirow{4}{*}{ MSSM RPV } & $d s+\bar{d} \bar{s}$ & $d \Delta s+s \Delta d+\bar{d} \Delta \bar{s}+\bar{s} \Delta \bar{d}$ & $\Delta d \Delta s+\Delta \bar{d} \Delta \bar{s}$ \\
& $d b+\bar{d} \bar{b}$ & $d \Delta b+b \Delta d+\bar{d} \Delta \bar{b}+\bar{b} \Delta \bar{d}$ & $\Delta d \Delta b+\Delta \bar{d} \Delta \bar{b}$ \\
& $s b+\bar{s} \bar{b}$ & $s \Delta b+b \Delta s+\bar{s} \Delta \bar{b}+\bar{b} \Delta \bar{s}$ & $\Delta s \Delta b+\Delta \bar{s} \Delta \bar{b}$ \\
\hline \multirow{3}{*}{ Hylogenesis } & $d d+\bar{d} \bar{d}$ & $d \Delta d+\bar{d} \Delta \bar{d}$ & $\Delta d \Delta d+\Delta \bar{d} \Delta \bar{d}$ \\
& $s s+\bar{s} \bar{s}$ & $s \Delta s+\bar{s} \Delta \bar{s}$ & $\Delta s \Delta s+\Delta \bar{s} \Delta \bar{s}$ \\
& $b b+\bar{b} \bar{b}$ & $b \Delta b+\bar{b} \Delta \bar{b}$ & $\Delta b \Delta b+\Delta \bar{b} \Delta \bar{b}$ \\
\hline \multirow{2}{*}{-model } & $g u+g \bar{u}$ & $g \Delta u+u \Delta g+g \Delta \bar{u}+\bar{u} \Delta g$ & $\Delta g \Delta u+\Delta g \Delta \bar{u}$ \\
& $g c+g \bar{c}$ & $g \Delta c+c \Delta g+g \Delta \bar{c}+\bar{c} \Delta g$ & $\Delta g \Delta c+\Delta g \Delta \bar{c}$ \\
\hline
\end{tabular}

Table 1. Parton luminosities that contribute to the unpolarized cross-section, the single- and the double-spin asymmetries in the three different scenarios for monotop production that are discussed in this paper. For each model, the first row corresponds to the dominant production channel. In singly-polarized collisions, the second hadron is the one that we choose to be polarized.

where $\hat{s}$ denotes the partonic center-of-mass energy and $h_{1}$ and $h_{2}$ the helicities of the initial antiquarks. Results for the charge-conjugate process can be obtained by replacing $h_{i} \rightarrow-h_{i}$. Moreover, we have assumed that only one of the two stop mass-eigenstates is light enough to significantly contribute to the cross-section and kept the associated dependence on the stop mixing angle $\theta_{\tilde{t}}$ explicit. Finally, we have adopted the narrowwidth approximation to model the resonant behavior of the squared matrix element by a Breit-Wigner lineshape. Although non-general, such an approximation holds when the width of the resonance is small with respect to its mass, which allows one to neglect offshell effects, when the resonance decays into much lighter particles and when its mass is much smaller than the center-of-mass energy, which avoids important distortions of the Breit-Wigner lineshape [40].

Because of the symmetry properties of the RPV superpotential of eq. (3.1), the couplings of quarks or antiquarks of the same flavor to the stop $\tilde{t}$ vanish so that only three different flavor combination can yield a non-zero cross-section, namely the $d s+\bar{d} \bar{s}, d b+\bar{d} \bar{b}$ and $s b+\bar{s} \bar{b}$ initial states. Here we are implicitly summing over both monotop and antimonotop production, while later in the section, we will explore the potential of tagging the charge of the final-state monotop. The parton luminosities that contribute to the unpolarized cross-section, the single- and the double-spin asymmetries in the various different scenarios for monotop production that are discussed in this paper are summarized in table 1.

In figure 7 , we present total cross-sections for RPV monotop production at the LHC $14 \mathrm{TeV}$ (left panel of the figure) and at an FCC $100 \mathrm{TeV}$ (right panel of the figure) as function of the mass of the lightest top squark. We compute our results by making use of eq. (2.14) with the NNPDF2.3 set of parton densities, and for the sake of the example, we consider maximal stop mixing $\left(\theta_{\tilde{t}}=\pi / 4\right)$, the branching ratio of the stop resonance into a monotop state equal to unity $\left(\operatorname{BR}\left(\tilde{t} \rightarrow t \tilde{\chi}_{1}^{0}\right)=1\right)$ and all the three possible different initial 



Figure 7. RPV monotop production total cross sections at the LHC $14 \mathrm{TeV}$ (left panel) and at an FCC $100 \mathrm{TeV}$ (right panel) as function of the invariant mass of the final state. We fix the stop mixing angle to $\pi / 4$, consider the branching ratio $\operatorname{BR}\left(\tilde{t} \rightarrow t \tilde{\chi}_{1}^{0}\right)=1$ and address three distinct benchmark scenarios where one single RPV coupling is non-vanishing at a time: $\lambda_{312}^{\prime \prime}$ (green), $\lambda_{313}^{\prime \prime}$ (blue) and $\lambda_{323}^{\prime \prime}$ (red). Cross-sections have been obtained using the NNPDF2.3 unpolarized parton set.

states. We however assume that only one of the three RPV couplings is non-zero at a time and that its value is fixed to $\lambda^{\prime \prime}=0.2$. With a quadratic dependence on the $\lambda^{\prime \prime}$-parameters, RPV monotop production could be in principle expected both at the LHC and at an FCC. However, characterizing which partonic initial state would be (dominantly) responsible for the possible observation of an excess is far more complicated than measuring a total crosssection. The standard approach would then be to probe differential distributions sensible, e.g., to the presence of valence or sea quarks in the initial state. However, in the rest of this section we focus on a complementary approach to characterize the initial state of monotop production by means of spin asymmetry measurements in polarized $p p$ collisions.

In the RPV context, there is only one single combination of quark helicities that gives rise to a monotop final state, as indicated in eq. (3.2). Consequently, partonic spin asymmetries turn out to be equal to \pm 1 and hadronic asymmetries reduce to ratios of partonic luminosities. Therefore, in the approximation in which there is a single dominant coupling $\lambda^{\prime \prime}$, hadron-level spin-asymmetries can be expressed in terms of a ratio of linear combinations of polarized and unpolarized PDFs and the results of section 2 hold. For instance, for the case of monotop production in the dominant channel $\bar{d} \bar{s}+d s$ we have

$$
A_{L}^{\bar{d} \bar{s}+d s}=\frac{\mathcal{L}_{d s}^{L}-\mathcal{L}_{\bar{d} \bar{s}}^{L}}{\mathcal{L}_{d s}+\mathcal{L}_{\bar{d} \bar{s}}} \quad \text { and } \quad A_{L L}^{\bar{d} \bar{s}+d s}=\frac{\mathcal{L}_{d s}^{L L}+\mathcal{L}_{\bar{d} \bar{s}}^{L L}}{\mathcal{L}_{d s}+\mathcal{L}_{\bar{d} \bar{s}}}
$$

and likewise for other initial states. We collect the results for the relevant channels in figure 8 for both the LHC $14 \mathrm{TeV}$ (left panel) and the FCC $100 \mathrm{TeV}$ (right panel), after summing over both monotop and anti-monotop production modes, and show single-spin (upper row of the figure) and double-spin (lower row of the figure) asymmetries.

It is clear from figure 8 that polarized asymmetries can be sizable, and moreover depend strongly on the partonic initial state. For instance, at the LHC $14 \mathrm{TeV}$ and $m_{X}=3 \mathrm{TeV}$, $A_{L}$ varies from $20 \%$ for the $s b$ initial state to $-30 \%$ for the $d s$ combination. The different behaviors of $A_{L L}$ and $A_{L}$ for the same partonic initial state has also discrimination power. 



Figure 8. Single-spin (upper panel) and double-spin (lower panel) asymmetries for RPV monotop production at the LHC $14 \mathrm{TeV}$ (left panel) and at an FCC $100 \mathrm{TeV}$ (right panel) as function of the stop (or monotop) mass. We fix the stop mixing angle to $\pi / 4$, consider the branching ratio $\operatorname{BR}\left(\tilde{t} \rightarrow t \tilde{\chi}_{1}^{0}\right)=1$ and address three distinct benchmark scenarios where one single RPV coupling is non-vanishing at a time: $\lambda_{312}^{\prime \prime}$ (green), $\lambda_{313}^{\prime \prime}$ (blue) and $\lambda_{323}^{\prime \prime}$ (red). Asymmetries have been obtained using NNPDFpol1.1 and NNPDF2.3.

Therefore, it can be seen that the availability of polarized beams at high energy hadron colliders allows to disentangle the different possible scenarios leading to monotop production, especially for large final-state masses, where the polarized asymmetries are larger.

\subsection{Other scenarios for monotop production}

In addition to the RPV MSSM scenario, several other models predict monotop production at hadron colliders. Therefore, in the event of observation of the monotop signature, determining which is the correct underlying model will be a difficult task. In particular, even disentangling a resonant monotop production from a non-resonant one might be nontrivial due to detector effects distorting typical resonant shapes expected, for instance, in the missing energy spectrum [15]. In section 3.1, we have investigated monotop production in the context of RPV supersymmetry and have shown how spin asymmetries could help characterizing the type of RPV interactions relevant for the production of a monotop state. We now investigate two additional scenarios predicting the production of a top quark in association with missing energy, and illustrate the strengths of measuring spin asymmetries in polarized collisions in order to obtain information on the underlying model. 


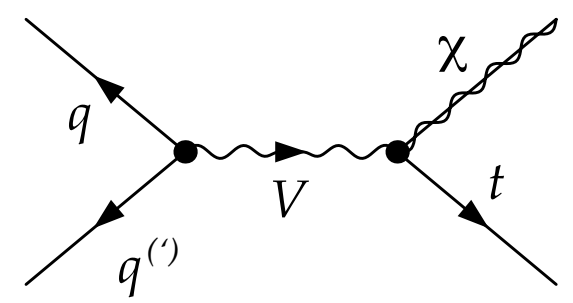

Figure 9. Feynman diagram associated with Hylogenesis monotop production.

We first focus on the so-called Hylogenesis models for dark matter where a monotop state can be produced from the decay of a heavy vector resonance $V_{\mu}$ of mass $m_{V}$ that couples to down-type quarks [20,21]. The leading order Feynman diagram for monotop production in this scenario is shown in figure 9 . The heavy vector resonance $V_{\mu}$ decays into an associated pair comprised of a top quark and a spin-1/2 dark matter particle, carrying missing energy, that we generically denote by $\chi$. We further describe the couplings of down-type quarks to the colored resonance $V_{\mu}$ with charge $\pm 2 / 3$ by the Lagrangian ${ }^{2}$

$$
\mathcal{L}_{\text {hylo }}=\frac{1}{2} \kappa_{i j} \bar{d}_{i}^{c} \gamma^{\mu} d_{j} V_{\mu}+\text { h.c. },
$$

where again a sum over color indices is understood, $i$ and $j$ are flavor indices and $\kappa_{i j}$ denotes the $3 \times 3$ (symmetric) matrix of interaction strengths in flavor space. As in eq. (3.2) for the RPV case, we will rewrite any dependence on the interactions of the dark matter state $\chi$ in terms of the branching ratio of $V_{\mu}$ to a monotop final state, so that the corresponding Lagrangian contributions are unnecessary and have been omitted. The constraints on the parameters of this scenario from collider and flavor physics have been studied in ref. [41], which shows that they are model dependent and can be easily avoided, specially in the case of third generation quarks. In the rest of this section, we restrict ourselves to the dominant $d d+\bar{d} \bar{d}$ production channel, again summing over monotop and anti-monotop production. As stated above, a discussion on the information that can be obtained by tagging the charge of the final-state top quark, and therefore disentangling monotop and anti-monotop production, will be carried out later in this section.

The third model for monotop production that we consider in this work will be denoted by the name ' $X$-Model'. It is motivated by models of dark matter where the top quark couples to a new neutral vector boson $X_{\mu}$ strongly interacting with invisible particles of a hidden sector $[22,23]$. The Feynman diagrams for monotop production in this scenario are shown in figure 10. In this case, the associated production of the new $X$-boson, which typically decays into particles of the hidden sector and thus escapes detection, with a top quark leads to a monotop signature. Adopting a simplified approach, we fix the part of the Lagrangian relevant for monotop production to

$$
\mathcal{L}_{X}=g_{X}^{i} \bar{u}_{i} \gamma^{\mu} P_{R} t X_{\mu}+\text { h.c. },
$$

\footnotetext{
${ }^{2}$ The Lagrangian choice is not unique and we focus on one particular example among others that induces large differences compared to the RPV case.
} 




Figure 10. Feynman diagrams associated with monotop production from flavor-changing interactions associated with an extra vector boson $X$ in the $X$-model.

where $P_{R}$ denotes the right-handed chirality projector and $g_{X}$ the associated vector of coupling constants in generation space. In the following, we focus on the dominant production channel where the $X$-boson couples to an up quark and a top quark (see table 1 ). The experimental constraints on the new physics mass scale in this scenario have been studied in ref. [22]. Their findings indicate that the mass of the $X$ field can be as low as $100 \mathrm{GeV}$ without conflicting with current bounds, such as $B_{d, s}-\bar{B}_{d, s}$ mixing or rare top decays.

The two Lagrangians of eqs. (3.4) and (3.5) allow us to calculate the corresponding fully polarized partonic cross-sections. We obtain, using the narrow width approximation for the Hylogenesis case and providing the differential cross-section with respect to the Mandelstam $t$-variable for the $X$-model,

$$
\begin{aligned}
& \hat{\sigma}_{\text {hylo }}^{h_{1} h_{2}}\left(\bar{q}_{j} \bar{q}_{k} \rightarrow t \chi\right)=\frac{2\left(1-h_{1} h_{2}\right) \pi\left|\kappa_{j k}\right|^{2}}{3} \times \mathrm{BR}(V \rightarrow t \chi) \times \delta\left(\hat{s}-m_{V}^{2}\right), \\
& \frac{\mathrm{d} \hat{\sigma}_{X}^{h, \lambda}}{\mathrm{d} t}\left(u_{i} g \rightarrow t X\right)=\frac{1}{16 \pi s^{2}} \frac{g_{s}^{2} g_{X}^{i 2}}{12 s m_{X}^{2}\left(t-m_{t}^{2}\right)^{2}}(1+h)\left[C_{1}+C_{2} \lambda\right] .
\end{aligned}
$$

In the Hylogenesis model, $h_{1}, h_{2}$ are the helicities of the initial partons and the results for the charge-conjugate processes are obtained by replacing $h_{i} \rightarrow-h_{i}$. It is clear from the fully polarized partonic cross-section of eq. (3.6) that in this case, the single-spin asymmetries vanish exactly. In contrast, for the double-polarized asymmetries, we have a similar situation as for RPV monotop production and the hadron-level asymmetries can be written in terms of ratios of the partonic luminosities discussed in section 2. To be explicit, for the dominant production channel $\bar{d} \bar{d}+d d$ we have

$$
A_{L}^{\bar{d} \bar{d}+d d}=0, \quad A_{L L}^{\bar{d} \bar{d}+d d}=-\frac{\mathcal{L}_{d d}^{L L}+\mathcal{L}_{\bar{d} \bar{d}}^{L L}}{\mathcal{L}_{d d}+\mathcal{L}_{\bar{d} \bar{d}}},
$$

and likewise for other initial states.

In the $X$-model calculation, we have kept the dependence on the gluon and initial quark polarizations $\lambda$ and $h$ explicit and have introduced the kinematical factors

$$
\begin{aligned}
C_{1}(s, t)= & m_{t}^{8}-m_{t}^{6}[2 s+t]+m_{t}^{4}\left[(s+t)^{2}-2 m_{X}^{2}\left(t+m_{X}^{2}\right)\right] \\
& +m_{t}^{2}\left[4 m_{X}^{6}-2 m_{X}^{4} t+2 m_{X}^{2}\left(s^{2}-s t+2 t^{2}\right)-t(s+t)^{2}\right] \\
& -2 m_{X}^{2} t\left(2 m_{X}^{4}+s^{2}+t^{2}-2 m_{X}^{2}(s+t)\right], \\
C_{2}(s, t)= & {\left[m_{t}^{4}+m_{t}^{2}\left(2 m_{X}^{2}-s-t\right)+2 m_{X}^{2}(s-t)\right]\left[m_{t}^{4}+t\left(2 m_{X}^{2}-s-t\right)-m_{t}^{2}\left(2 m_{X}^{2}+s\right)\right] . }
\end{aligned}
$$



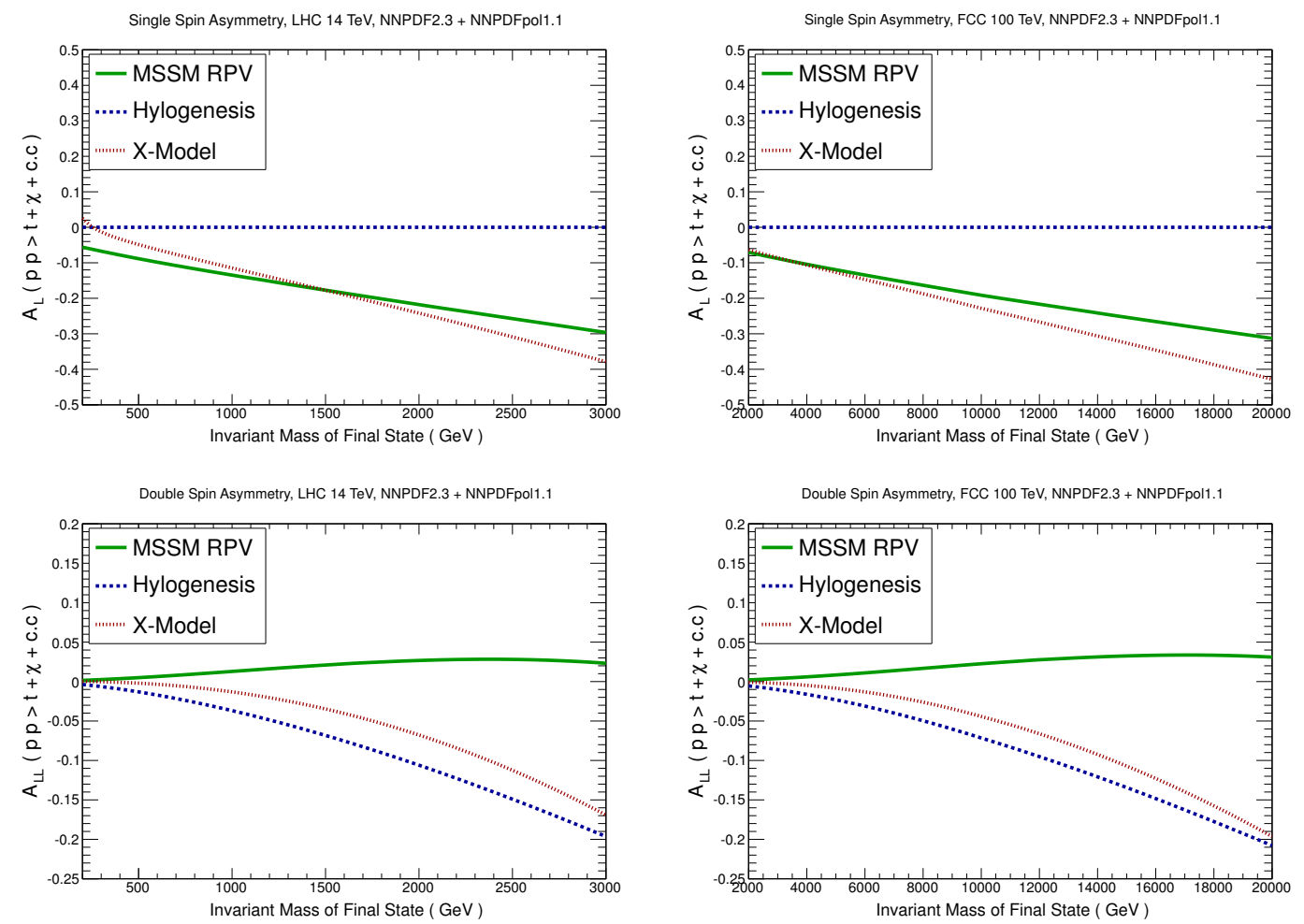

Figure 11. Single-spin (upper panel) and double-spin (lower panel) asymmetries for monotop production at the LHC $14 \mathrm{TeV}$ (left panel) and at an FCC $100 \mathrm{TeV}$ (right panel) as function of the monotop system mass for the various new physics scenarios described in the text. Asymmetries have been obtained using NNPDFpol1.1 and NNPDF2.3. Sum over monotop and anti-monotop production is implicit.

The main feature of this class of models with respect to the RPV case lies in the various helicity combinations contributing to the polarized cross-section. Important differences are consequently expected in spin asymmetries when comparing RPV monotop production to Hylogenesis or dark matter $X$-model predictions.

This is illustrated in figure 11 where we compare, for illustrative purposes, single-spin and double-spin asymmetries as predicted in RPV scenarios where the monotop system originates from a $d s+\bar{d} \bar{s}$ initial state, in Hylogenesis models where it is produced from $d d+\bar{d} \bar{d}$ scattering and in dark matter $X$-models where the $X$-boson arises from the $g(u+\bar{u})$ initial state. We present our results as functions of the monotop system mass being defined as the resonance mass for both the RPV and the Hylogenesis scenarios, and as the sum of the $X$-boson and top quark masses for the $X$-model case. ${ }^{3}$

Figure 11 is the main result of this work. It tells us that, assuming polarized PDF uncertainties are improved by a series of dedicated measurements, a measurement of the single-spin asymmetry with $5 \%$ precision would allow one to discriminate between the three production mechanisms for states with sufficiently large invariant mass, approximately

\footnotetext{
${ }^{3}$ The numerical values of all other model parameters are irrelevant as canceling in the ratios of polarized and unpolarized cross-sections.
} 
above $2 \mathrm{TeV}$ at the LHC and above $10 \mathrm{TeV}$ at the FCC. Even a measurement of the sign of the single spin asymmetries would be very valuable to discriminate between different scenarios. Double-spin asymmetries would provide a complementary cross-check of the single-spin results, though their measurement is rather more challenging both because of the reduced rates and because of the smaller values of the asymmetries. The qualitative behavior of $A_{L}$ and $A_{L L}$ is also found to be rather different in some scenarios. In RPV monotop production, for instance, $A_{L}$ is large and negative, while $A_{L L}$ is small and positive. It is thus clear that a simultaneous measurement of $A_{L}$ and $A_{L L}$ would provide stringent constraints on the underlying production dynamics.

\subsection{Impact of monotop charge tagging}

In the final part of this section, we study what we can learn if the charge of the final-state top quark is tagged, that is, if we are able to disentangle the monotop signature (a top quarks of charge $+2 / 3$ and missing transverse energy) from the anti-monotop signature (same with a top antiquark). This charge tagging could be potentially relevant because in these two cases, the polarized PDFs that are relevant according to the nature of the initial state can show quite different behaviors. Tagging the charge of the top quark can thus provide another handle on the underlying BSM scenario that has induced monotop production. ${ }^{4}$

We show in figure 12 the single-spin asymmetries for LHC $14 \mathrm{TeV}$ and FCC $100 \mathrm{TeV}$, this time separating monotop from anti-monotop production, for the three models under consideration. The relevant initial states for monotop production are $\bar{d} \bar{s}, \bar{d} \bar{d}$ and $u g$ in the RPV, Hylogenesis and $X$-model scenarios respectively, and the corresponding chargeconjugate ones for anti-monotop production. It is clear from the differences between the left and right columns of figure 12 that tagging the top quark charge provides important information about the underlying production model, with the differences particularly striking in the case of the RPV scenario, where at large masses a different sign of the asymmetry is predicted in the two cases.

We recall that the results of figure 11 cannot be retrieved by a trivial average of the asymmetries of figure 12 over monotop and anti-monotop production, as the full singly-polarized and unpolarized cross sections need to be averaged first, before evaluating the ratios.

\subsection{Summary}

In this section, we have shown how, using polarized collisions, it is possible to discriminate between different models that lead to the same final-state signature, in this case monotop production. While we have considered this specific benchmark scenario, it is clear that our results/our considerations apply to a wide variety of other BSM models where the availability of polarized beams would provide a unique handle for their characterization.

\section{Conclusions and outlook}

In this paper, we have motivated how the availability of polarized beams at high-energy hadron colliders provides a unique handle on the discrimination between different beyond

\footnotetext{
${ }^{4}$ Charge asymmetries are also interesting observables in the context of unpolarized collisions.
} 

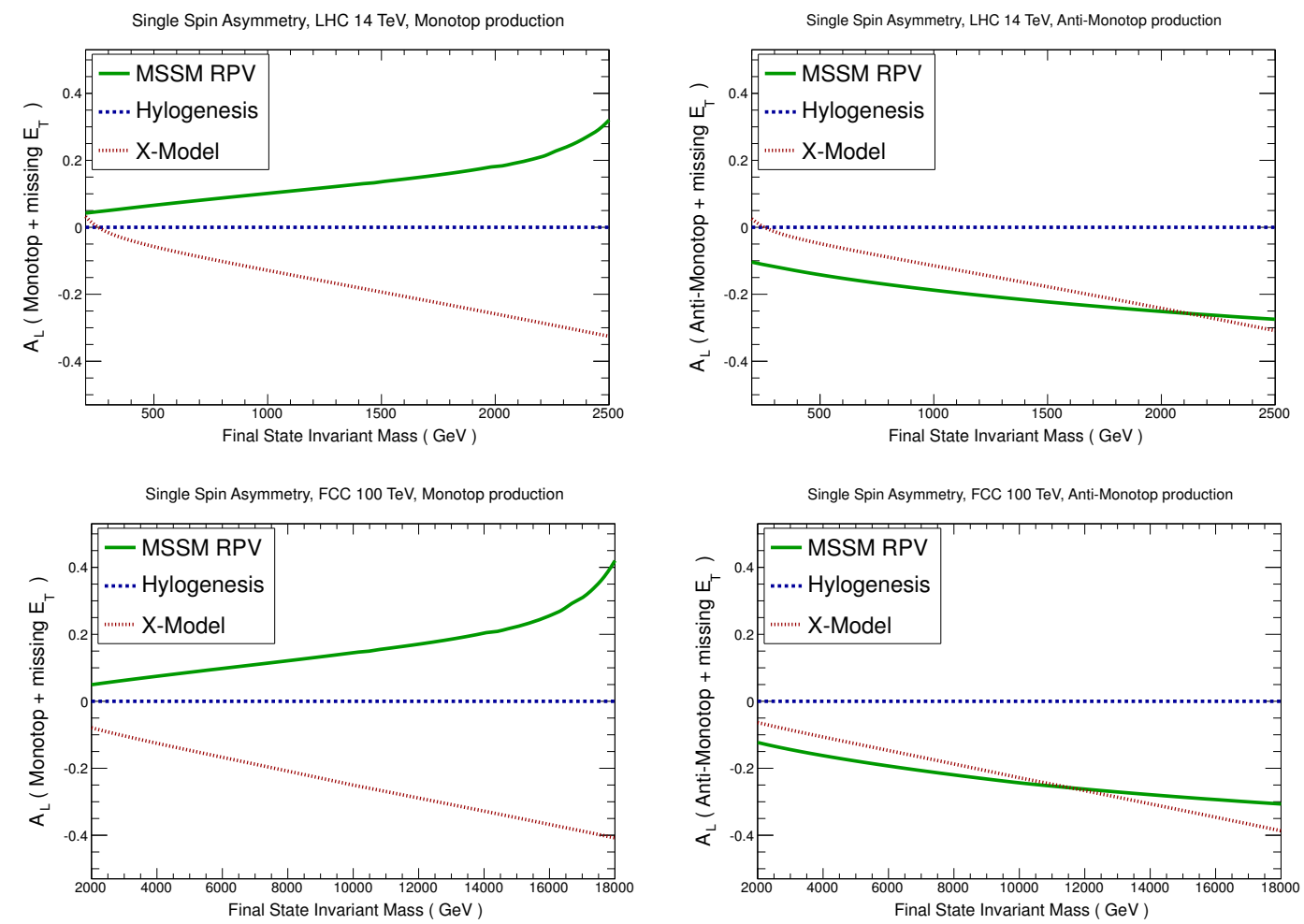

Figure 12. Single-spin asymmetries for monotop (left panel) and anti-monotop (right panel) production at the LHC $14 \mathrm{TeV}$ (upper row) and at an FCC $100 \mathrm{TeV}$ (lower row) as function of the invariant mass of the final state for the different new physics scenarios discussed in this work.

the Standard Model scenarios that lead to the same final-state signatures in unpolarized collisions. First of all, we have discussed in a model-independent way why single and double-spin asymmetries in polarized collisions allow us for the separation between different initial-state production mechanisms. Then we have considered different benchmark scenarios for monotop production and shown how the measurement of spin asymmetries in polarized collisions could help to discriminate between different models. Therefore, while polarized beams are certainly not required for BSM discoveries, they can provide very useful information on the properties of the hypothetical BSM sector, in particular in the determination of its couplings to Standard Model particles.

While technically feasible, the likelihood of a future polarized mode at the LHC is very small, requiring a complete modification of the full injector chain. The situation might however be different for the recently proposed Future Circular Collider (FCC) at a center-of-mass energy of $100 \mathrm{TeV}$ : if there is a strong physics case, we believe that the polarized option should be considered seriously. In any case this is the right time to begin to think of the feasibility of such an option, now that various studies for the planning of this machine have just started. In particular, if new physics is discovered at the LHC during the proton-proton runs at center-of-mass energies of $13 \mathrm{TeV}, 14 \mathrm{TeV}$ or at the future high-luminosity upgrade of the LHC, there will be a very strong motivation for a polarized mode of the FCC in order to characterize and understand the properties of this new sector. 
Future studies, along similar directions as the ones we have explored in this paper, should be performed in two different and complementary directions. On the one hand, other BSM scenarios should be studied. These studies should focus on the production of highmass particles, since as we discussed this is the only region where single- and double-spin asymmetries are relatively large and thus experimentally accessible. A possible example would be to estimate the accuracy to which the couplings of a possible heavy $Z^{\prime}$ can be determined at the FCC from polarized collisions. On the other hand, one also needs to perform more detailed feasibility studies for the measurement of single- and double-spin asymmetries, trying to estimate the luminosities in the polarized mode that a $100 \mathrm{TeV}$ FCC could deliver and how the rates would be affected by the finite polarization of the beams. Quantifying the statistical uncertainties of the spin asymmetries at the FCC would also allow one to better understand what is the reach of BSM characterization of the polarized collision mode.

As an intriguing final remark, it should be noted that at a $100 \mathrm{TeV}$ FCC it might be possible to access polarized collisions without the need of using polarized beams. ${ }^{5}$ Indeed, at the scale of $10-20 \mathrm{TeV}$, the electroweak $W$ - and $Z$-bosons are effectively massless and should be included in the DGLAP evolution, which leads at this point to an intrinsic polarization of the quarks and gluons via mixing. This is an interesting possibility to study further, since in any case PDFs with electroweak corrections are mandatory for the physics of a $100 \mathrm{TeV}$ hadron collider.

\section{Acknowledgments}

The authors are grateful to Emanuele Nocera for useful discussions. This work has been partially supported by the Théorie-LHC-France initiative of the CNRS/IN2P3, by the French ANR 12 JS05 00201 BATS@LHC and by a PhD grant of the 'Investissements d'avenir, Labex ENIGMASS'.

Open Access. This article is distributed under the terms of the Creative Commons Attribution License (CC-BY 4.0), which permits any use, distribution and reproduction in any medium, provided the original author(s) and source are credited.

\section{References}

[1] H.P. Nilles, Supersymmetry, supergravity and particle physics, Phys. Rept. 110 (1984) 1 [INSPIRE].

[2] H.E. Haber and G.L. Kane, The search for supersymmetry: probing physics beyond the standard model, Phys. Rept. 117 (1985) 75 [INSPIRE].

[3] T. Appelquist, H.-C. Cheng and B.A. Dobrescu, Bounds on universal extra dimensions, Phys. Rev. D 64 (2001) 035002 [hep-ph/0012100] [INSPIRE].

[4] N. Craigie, K. Hidaka and P. Ratcliffe, The role helicity asymmetries could play in the search for supersymmetric interactions, Phys. Lett. B 129 (1983) 310 [InSPIRE].

\footnotetext{
${ }^{5}$ We thank G. Salam for pointing out this observation to us.
} 
[5] P. Taxil and J. Virey, Discovery limits for a new contact interaction at future hadronic colliders with polarized beams, Phys. Rev. D 55 (1997) 4480 [hep-ph/9607390] [INSPIRE].

[6] J. Virey, Hunting for contact interactions at HERA with polarized lepton and proton beams, Eur. Phys. J. C 8 (1999) 283 [hep-ph/9809439] [INSPIRE].

[7] P. Taxil, E. Tugcu and J. Virey, Constraints on leptophobic gauge bosons with polarized neutrons and protons at RHIC, Eur. Phys. J. C 24 (2002) 149 [hep-ph/0111242] [INSPIRE].

[8] T. Gehrmann, D. Maître and D. Wyler, Spin asymmetries in squark and gluino production at polarized hadron colliders, Nucl. Phys. B 703 (2004) 147 [hep-ph/0406222] [INSPIRE].

[9] G. Bozzi, B. Fuks and M. Klasen, Slepton production in polarized hadron collisions, Phys. Lett. B 609 (2005) 339 [hep-ph/0411318] [INSPIRE].

[10] J. Debove, B. Fuks and M. Klasen, Model-independent analysis of gaugino-pair production in polarized and unpolarized hadron collisions, Phys. Rev. D 78 (2008) 074020 [arXiv: 0804.0423] [INSPIRE].

[11] M. Wiest, D.R. Stump, D.O. Carlson and C. Yuan, Studying anomalous $W W \gamma$ and $W W Z$ couplings with polarized pp collisions, Phys. Rev. D 52 (1995) 2724 [hep-ph/9506327] [INSPIRE].

[12] SPIN collaboration, R. Baiod, P. Martin, and A. Russell, Polarized protons in the Tevatron collider, contribution to the $10^{\text {th }}$ Topical Workshop on Proton-Antiproton Collider Physics, May 9-13, Batavia, Illinois, U.S.A. (1995).

[13] A. De Roeck, D. Barber and G. Radel, Polarized protons at high-energies, in the proceedings of the DESY workshop on Polarized Protons at High Energies, May 17-20, Hamburg, Germany (1999).

[14] J. Andrea, B. Fuks and F. Maltoni, Monotops at the LHC, Phys. Rev. D 84 (2011) 074025 [arXiv: 1106.6199] [INSPIRE].

[15] J.-L. Agram, J. Andrea, M. Buttignol, E. Conte and B. Fuks, Monotop phenomenology at the Large Hadron Collider, Phys. Rev. D 89 (2014) 014028 [arXiv: 1311.6478] [InSPIRE].

[16] E.L. Berger, B. Harris and Z. Sullivan, Single top squark production via R-parity violating supersymmetric couplings in hadron collisions, Phys. Rev. Lett. 83 (1999) 4472 [hep-ph/9903549] [INSPIRE].

[17] E.L. Berger, B. Harris and Z. Sullivan, Direct probes of R-parity violating supersymmetric couplings via single top squark production, Phys. Rev. D 63 (2001) 115001 [hep-ph/0012184] [INSPIRE].

[18] N. Desai and B. Mukhopadhyaya, R-parity violating resonant stop production at the Large Hadron Collider, JHEP 10 (2010) 060 [arXiv: 1002.2339] [INSPIRE].

[19] B. Fuks, Beyond the minimal supersymmetric standard model: from theory to phenomenology, Int. J. Mod. Phys. A 27 (2012) 1230007 [arXiv:1202.4769] [INSPIRE].

[20] H. Davoudiasl, D.E. Morrissey, K. Sigurdson and S. Tulin, Hylogenesis: a unified origin for baryonic visible matter and antibaryonic dark matter, Phys. Rev. Lett. 105 (2010) 211304 [arXiv: 1008.2399] [INSPIRE].

[21] H. Davoudiasl, D.E. Morrissey, K. Sigurdson and S. Tulin, Baryon destruction by asymmetric dark matter, Phys. Rev. D 84 (2011) 096008 [arXiv:1106.4320] [INSPIRE].

[22] J.F. Kamenik and J. Zupan, Discovering dark matter through flavor violation at the LHC, Phys. Rev. D 84 (2011) 111502 [arXiv:1107.0623] [InSPIRE].

[23] E. Alvarez, E.C. Leskow, J. Drobnak and J.F. Kamenik, Leptonic monotops at LHC, Phys. Rev. D 89 (2014) 014016 [arXiv: 1310.7600] [InSPIRE]. 
[24] R.D. Ballet al., Parton distributions with LHC data, Nucl. Phys. B 867 (2013) 244 [arXiv: 1207.1303] [INSPIRE].

[25] NNPDF collaboration, R.D. Ball et al., Unbiased determination of polarized parton distributions and their uncertainties, Nucl. Phys. B 874 (2013) 36 [arXiv:1303.7236] [INSPIRE].

[26] E.R. Nocera, Unbiased spin-dependent parton distribution functions, arXiv: 1403.0440 [INSPIRE].

[27] M. Whalley, D. Bourilkov and R. Group, The Les Houches accord PDFs (LHAPDF) and LHAGLUE, hep-ph/0508110 [INSPIRE].

[28] G. Altarelli, S. Forte and G. Ridolfi, On positivity of parton distributions, Nucl. Phys. B 534 (1998) 277 [hep-ph/9806345] [INSPIRE].

[29] R.D. Ball, S. Forte and G. Ridolfi, Scale dependence and small x behavior of polarized parton distributions, Nucl. Phys. B 444 (1995) 287 [Erratum ibid. B 449 (1995) 680] [hep-ph/9502340] [INSPIRE].

[30] J.M. Campbell, J. Huston and W. Stirling, Hard interactions of quarks and gluons: a primer for LHC physics, Rept. Prog. Phys. 70 (2007) 89 [hep-ph/0611148] [InSPIRE].

[31] D. de Florian, R. Sassot, M. Stratmann and W. Vogelsang, Extraction of spin-dependent parton densities and their uncertainties, Phys. Rev. D 80 (2009) 034030 [arXiv:0904.3821] [INSPIRE].

[32] A.D. Martin, R. Roberts, W. Stirling and R. Thorne, MRST2001: partons and $\alpha_{s}$ from precise deep inelastic scattering and Tevatron jet data, Eur. Phys. J. C 23 (2002) 73 [hep-ph/0110215] [INSPIRE].

[33] The NNPDF collaboration, R.D. Ball et al., Theoretical issues in PDF determination and associated uncertainties, Phys. Lett. B 723 (2013) 330 [arXiv:1303.1189] [INSPIRE].

[34] D. Boer et al., Gluons and the quark sea at high energies: distributions, polarization, tomography, arXiv:1108.1713 [INSPIRE].

[35] NNPDF collaboration, R.D. Ball et al., Polarized parton distributions at an electron-ion collider, Phys. Lett. B 728 (2014) 524 [arXiv:1310.0461] [INSPIRE].

[36] E.C. Aschenauer, T. Burton, T. Martini, H. Spiesberger and M. Stratmann, Prospects for charged current deep-inelastic scattering off polarized nucleons at a future electron-ion collider, Phys. Rev. D 88 (2013) 114025 [arXiv:1309.5327] [InSPIRE].

[37] E.C. Aschenauer, R. Sassot and M. Stratmann, Helicity parton distributions at a future electron-ion collider: a quantitative appraisal, Phys. Rev. D 86 (2012) 054020 [arXiv:1206.6014] [INSPIRE].

[38] R. Barbier et al., R-parity violating supersymmetry, Phys. Rept. 420 (2005) 1 [hep-ph/0406039] [INSPIRE].

[39] R parity Working Group collaboration, B. Allanach et al., Searching for R parity violation at Run II of the Tevatron, hep-ph/9906224 [INSPIRE].

[40] D. Berdine, N. Kauer and D. Rainwater, Breakdown of the narrow width approximation for new physics, Phys. Rev. Lett. 99 (2007) 111601 [hep-ph/0703058] [INSPIRE].

[41] I.-W. Kim and K.M. Zurek, Flavor and collider signatures of asymmetric dark matter, Phys. Rev. D 89 (2014) 035008 [arXiv:1310.2617] [INSPIRE]. 